\title{
Designing Automated Handheld Navigation Support
}

\author{
Doguhan Uluca ${ }^{1}$, Jan Willem Streefkerk ${ }^{2}$, Brian Sciacchitano ${ }^{3}$, D. Scott McCrickard ${ }^{3}$ \\ ${ }^{1}$ Product Development \\ Meridium, Inc. \\ Roanoke, VA 24018, USA \\ $+1(540) 3449205$ \\ duluca@meridium.com \\ ${ }^{3}$ Department of Computer Science
\& Center for HCl
Virginia Tech
Blacksburg, VA 24060, USA
+1 (540) 2316698
\{bsciacch; mccricks\}@vt.edu
}

\begin{abstract}
Map usage on handheld devices suffers from limited screen size and the minimal attention that users can dedicate to them in mobile situations. This work examines effects of automating navigation features like zooming and panning as well as other features such as rotation, path finding and artifact representation on the mobile navigation experience. Described are five claims and early support for these features in the police work domain.
\end{abstract}

\section{Categories and Subject Descriptors}

H.1.2 [Information Systems]: User/Machine Systems - Human factors, Human information processing.

H.5.2 [Information Systems]: User Interfaces - Graphical user interfaces (GUI), Screen design Interaction styles, Prototyping, User-centered design.

\section{General Terms}

Performance, Design, Experimentation, Human Factors.

\section{Author Keywords}

Navigation aids, Automated user interaction, Adaptive user interfaces, Mobile devices, Police, Loosely-directed navigation.

\section{Introduction}

This research seeks to explore any correlation between the type of task and level of automation in handheld navigation to better support task performance for mobile workers. This is especially necessary for mobile users in critical domains, such as security officers, emergency response and utility repair units. However, automation should support the user. Thus, design choices regarding which features should be automated must be based on the type of task a user needs to perform [3]. Using an established framework, IRC, we aim to investigate suitability of our claims in the work domain of mobile security officers.

\section{Navigation support}

Navigation support in police cars has become widespread, and here the need for proper design of these support systems is evident

Copyright is held by the author/owner(s).

MobileHCI 2008, September 2-5, 2008, Amsterdam, the Netherlands.

ACM ISBN 978-1-59593-952-4/08/09.
[1]. As of yet, no prototypes of navigation support systems for police officers on foot have been described in the literature [4]. The need for and benefits of navigation support while on foot have primarily been demonstrated in other domains such as tourism, military and urban search and rescue domains [5]. Based on domain research and interviews with police officers, we distinguished three generic navigation task types in the police work domain:

- Directed navigation: Based on an occurring (often highpriority) incident, police officers make their way as fast as possible to the incident location.

- "Loosely-directed" navigation: During a surveillance round, police officers visit certain checkpoints, such as crime hotspots. They can decide for themselves when and in what order to visit these points.

- Free navigation: Police officers walking their surveillance round must pay attention to safety in the streets and notice incidents or criminal circumstances wherever they occur. In this case, they may not have a destination they want to reach.

When navigation is not supported, problems can arise, such as missing or overshooting locations or hot-spots, inability to find a specific location, taking a wrong or inefficient route, and making general navigation errors. Task performance criteria can be defined for each type of task. In directed navigation, navigation speed and speed of response are all important. However, for the free task, the number of correctly identified incidents is important. Using the Interruption-Reaction-Comprehension (IRC) framework by McCrickard et al [2], we aim to model the appropriateness of our automated navigation features to support different types of tasks. In our model, Interruption should be low for Directed, medium for Loosely-directed and high for Free tasks. Reaction should be high for Directed and medium for Loosely-directed and Free tasks. Finally, Comprehension should be low for Directed and high for Loosely-directed and Free tasks.

\section{Navigation Features}

Analyzing the literature on mobile navigation resulted in identification of five core navigation features. Zooming, the ability to magnify or shrink a map; panning, the ability to dragand-release a map to glance at the surrounding area; rotation, the ability to modify the heading of a map; path finding or preservation, determining the shortest or fastest path between two points or preserving the integrity of predetermined routes; and artifact representation, disabling or enabling representations of real world objects or landmarks overlaid on the map. Zooming, panning and rotation features are powerful and necessary navigation features for small screen displays. They are intuitive as 
well as familiar to most users and do not clutter the user interface. Path finding or preservation and artifact representation features assist in locating off-screen or onscreen landmarks or artifacts.

\section{Claims}

Using these core features, we derived automated navigation support claims. However, not every claim is suitable for every type of task. Below, we present our claims and indicate for each claim whether it might be suitable for a Directed, Looselydirected or Free task respectively. ' + ' is used for very suitable, ' $+/-$ ' for somewhat suitable and '-' for not suitable.

Auto-zoom (+, +/-, -): Zooming in maps gives a better sense of orientation and control of the amount of information that the user is exposed to. However, the user needs to constantly manipulate the map to make better decisions about their environment. By automation zooming, the UI automatically changes the zoom level of the map so that the user and the destination are always displayed on the screen. This gives users flexibility in choosing their paths and ability to easily determine their location in relation to their destination. A glance of the user every once in a while conveys most of the information they need. See Figure 1.

Auto-pan (-, +/-, +/-): Panning promotes intuitive direct and free manipulation, but users must constantly interact with the map to use this feature. By automating panning, the UI automatically pans the map to keep the user in the center of the screen. This feature is most useful to users unfamiliar to their environment, because they are provided with a direction to their destination by an arrow. See Figure 2.

Auto-rotate $(+,+,+/-)$ : The user interface automatically rotates the map to match users' orientation and heading in the real world. This makes it easier for users to match routes and artifacts on the map to landmarks in the real world.

Path Visualization (+, +/-, -): The fastest or shortest route is indicated as a colored line between user's location and destination. This route automatically updates as users change their location, resulting in less navigational and mental strain on the user. The user can also manipulate the route in the display.

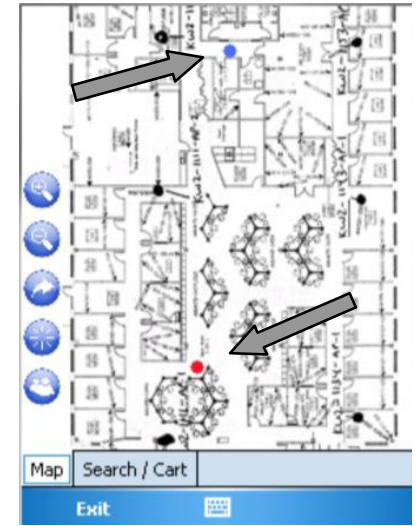

Figure 1. Auto-zoom feature. The red dot* (lower) denotes user's current location and the blue dot* (top) denotes user's destination.

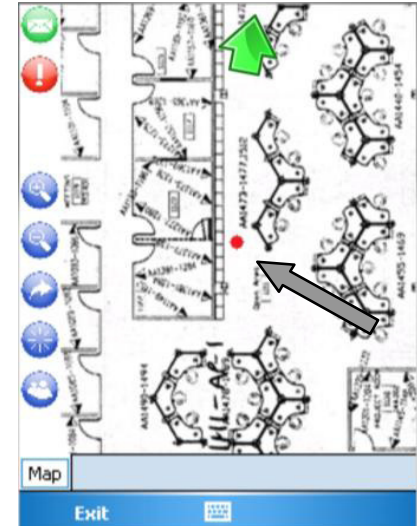

Figure 2. Auto-pan feature. The red dot* (center) denotes the user's current location. *Dots are pointed out by the thin arrows.
Artifact Presentation (+/-, +/-, +/-): The user interface changes the amount and nature of artifacts that are overlaid on the map at any given time. For example, the user interface could filter nonrelevant artifacts, or present a stylized map versus a realistic map. Although some relevant information may be blocked, users' focus on the task is maintained.

These claims can be compounded in various ways to increase or fine tune their suitability to a task. For example, consider a handheld device with all the automated features. In such a situation, security officers performing their rounds (a looselydirected task) would experience auto-pan, auto-rotation and artifact presentation. This would minimize user interaction and maximize artifact visibility, discoverability and as a result situational awareness. However, if an incident occurs nearby, the task type changes from loosely-directed to directed task. Subsequently, the interface changes to a combination of auto-zoom, auto-rotate and path visualization. The user would see the fastest route to the incident location, while his heading and distance determine the zoom level. This way, the features help the security officer get to the incident location as effectively as possible, while revealing greater detail and more information as the officer closes in on the destination.

\section{Conclusion \& Future Work}

To be able to test the validity of our predictions we plan to implement prototypes of our claims and test them with domain experts as well as everyday users. This will enable us to collect valuable suggestions from domain experts and test the intuitiveness of our designs with inexperienced users. The use of IRC questionnaires helps to determine the actual IRC scores for our features. These values will be compared with our IRC model to determine the validity of our claims. In doing this, the appropriateness of our automated handheld navigation support features can be optimized.

\section{References}

[1] Marcus, A. and Gasperini, J. 2006. Almost dead on arrival: A case study of non-user-centered design for a police emergency-response system. Interactions 13(5), 12-18.

[2] McCrickard, D. S., Chewar, C. M., Somervell, J. P. and Ndiwalana, A. 2003. A Model for Notification Systems Evaluation-Assessing User Goals for Multitasking Activity. ACM TOCHI 10(4), 312-338.

[3] Reichenbacher, T. 2004. Mobile Cartography - Adaptive Visualisation of Geographic Information on Mobile Devices. Doctoral Dissertation, University of Munich. Available online: http://tumb1.biblio.tu-muenchen.de/publ/diss/ bv/2004/reichenbacher.pdf

[4] Streefkerk, J.W., van Esch-Bussemakers, M.P. and Neerincx, M.A. 2006. Designing Personal Attentive User Interfaces in the Mobile Public Safety Domain. Computers in Human Behavior 22, 749-770.

[5] Te Brake, G., de Greef, T., Lindenberg, J., Rypkema, J. and Smets, N. 2006. Developing adaptive user interfaces using a game-based simulation environment. In Proceedings of ISCRAM 2006. 